\title{
Familial Intracranial Aneurysms
}

\author{
Jin Soo Lee, M.D., In Sung Park, M.D., Kyung Bum Park, M.D., Dong-Ho Kang, M.D., Chul Hee Lee, M.D., Soo Hyun Hwang, M.D. \\ Department of Neurosurgery, Gyeongsang National University School of Medicine, Jinju, Korea
}

\begin{abstract}
Objective : Numerous studies have compared the characteristics of familial intracranial aneurysms with those of non-familial aneurysms. To better understand familial subarachnoid hemorrhage (SAH), we studied a series of patients with SAH who had at least one first-degree relative with $\mathrm{SAH}$, and compared our results with those of previous studies.

Methods : We identified patients treated for SAH at our hospital between January 1993 and October 2006 and analyzed those patients with one or more first-degree relatives with SAH. We retrospectively collected data from patients with a family history and searched for patients who had relatives with aneurysms or who had been treated at other hospitals for SAH.

Results : We identified 12 patients from six families with at least two first-degree relatives with SAH. All patients had affected first-degree relatives; in five families, they were siblings. The mean age at the time of rupture was 49.75 years; in four families, the age difference was within 5 years. In five patients (42\%), the aneurysm was located in the middle cerebral artery. Only one patient had an aneurysm in the anterior communicating artery.

Conclusion : In agreement with previous studies, our results showed that familial aneurysms, in comparison with non-familiar aneurysms, ruptured at a younger age and smaller size, had a high incidence in the middle cerebral artery, and were underrepresented in the anterior communicating artery. Interestingly, the age at the time of rupture was similar between relatives. Screening should be considered in the fifth or sixth decade for those who have a sibling with $\mathrm{SAH}$.
\end{abstract}

KEY WORDS : Subarachnoid hemorrhage · Familial · Aneurysm · Screening.

\section{INTRODUCTION}

Recently, the incidence of cerebrovascular disease in Western countries has been decreasing, owing to the successful control of hypertension. Nevertheless, in Korea, cerebrovascular disease remains the second most common cause of death, following cancer, and subarachnoid hemorrhage (SAH) is the third major type of stroke ${ }^{13)}$.

SAH affects 8-10 per 100,000 persons per year ${ }^{4,18)}$. Most SAHs are attributable to the rupture of an intracranial aneurysm, and $25-50 \%$ of those patients die as a result ${ }^{6,18)}$. The etiology and pathogenesis of intracranial aneurysms are clearly multifactorial, with acquired (environmental) factors playing a major role. Many studies have found that genetic factors are also important ${ }^{23)}$. The two main lines of evidence are the association of intracranial aneurysm with heritable connective tissue disorders and the familial pattern

- Received : April 11, 2008 •Accepted : August 10, 2008

- Address for reprints : In Sung Park, M.D.

Department of Neurosurgery, Gyeongsang National University School

of Medicine, 90 Chiram-dong, Jinju 660-751, Korea

Tel : +82-55-750-8018, Fax : +82-55-759-0817

E-mail : gnuhpis@nongae.gsnu.ac.kr of intracranial aneurysms $\mathrm{s}^{19,20)}$.

The familial aggregation of intracranial aneurysms was first described in 1954 by Chambers and colleagues in a report on the cases of a father and son who each had an intracranial aneurysm ${ }^{5)}$. Numerous studies comparing the characteristics of familial intracranial aneurysms with those of non-familial (sporadic) aneurysms have found that familial aneurysms rupture about 5 years earlier, on average, and are smaller than sporadic aneurysms; are underrepresented in the anterior communicating artery; and frequently occur as multiple aneurysms $s^{4,711,19)}$. To compare the characteristics of familial SAH in our community with those reported in previous studies, we evaluated a series of patients with SAH who had at least one first-degree relative with SAH.

\section{MATERIALS AND METHODS}

We identified patients treated for SAH at our hospital between January 1993 and December 2006. Patients with perimesencephalic nonaneurysmal $\mathrm{SAH}$ or traumatic $\mathrm{SAH}$ were excluded. Intracranial aneurysms were identified by three-dimensional computed tomography or cerebral 
angiography. All patients with identified aneurysms had surgery or endovascular treatment. We retrospectively collected data from patients with a familial history of SAH and searched for patients who had relatives with aneurysms or who had been treated at other hospitals for SAH. All first-degree relatives (parents, children, and siblings) were interviewed by telephone, and their medical records were reviewed. Patients with one or more first-degree relatives with SAH were designated as having familial SAH. Patients with no relatives who experienced $\mathrm{SAH}$ were designated as the sporadic group. To understand the characteristics of familial SAH, we analyzed the multiplicity, size, and location of the aneurysms, as well as the age, gender, initial mental status, outcome, and past medical history of the patients. A pedigree was drawn for each family.

\section{RESULTS}

We operated on 1,128 SAH patients at our hospital over the 13 years examined in this study. We identified 13 patients from six families with at least two first-degree relatives with SAH. We excluded the mother in family 3 of the familial group because we could not confirm the images and results; we were aware of her SAH only from a statement by family members, as she had received treatment at another hospital. We excluded one patient in the familial group, leaving 12 patients from six families as the familial group. Table 1 shows the patient information. The primary way in which we discovered that patients had relatives with a history of SAH who had received treatment at our hospital was by taking the family medical history on admission. This might have been a consequence of regional characteristics, as many relatives live nearby and most local residents with cerebral vascular disease received therapy at our hospital. All patients in the familial group underwent operations within 4 days of their ruptured aneurysm. The remaining unruptured aneurysms in families 1, 4, and 6 received endovascular treatment, or an operation within 2 weeks. Patients received regular follow-up at our outpatient clinic.

There were several differences between the familial and sporadic groups. Table 2 shows the characteristics of the familial group. There was no remarkable difference in gender distribution in the familial SAH group, whereas females were dominant in the sporadic group. In the familial group, the mean age at the time of rupture was 49.75 years (range, 33-68 years), which was younger than that in the sporadic group (54.9 years). In the sporadic group, the most common site of aneurysm was the anterior communicating artery (33\%), followed by the middle cerebral artery $(24 \%)$, the internal cerebral artery (18\%), and multiple aneurysms (17\%). The familial group had a different site distribution : the most common location of aneurysm was the middle cerebral artery $(5 / 12,42 \%)$, followed by the internal cerebral artery $(3 / 12,25 \%)$. In the familial group, only one aneurysm was located in the anterior communicating artery, whereas that was the most common site in the sporadic group.

Multiple aneurysms were more common in the familial group than in the sporadic group. A son in family 1 had three aneurysms with mirror-image symmetry (anterior communicating artery and both posterior communicating arteries); a brother in family 4 had two aneurysms (middle cerebral artery and ophthalmic artery); and a brother in family 6 had three aneurysms (anterior cerebral artery, anterior communicating artery, and posterior communicating artery). The size of the ruptured aneurysm was generally small in both groups. In the familial group, nine patients had a small aneurysm $(<10 \mathrm{~mm})$ and two had a large aneurysm (10-24 mm); the size was unknown in one patient. The initial mental state of most patients in the familial group was good, ranging from mild-drowsy to alert; however, one patient was in a coma. All patients received emergency operations (craniotomy and direct neck clamping). One patient suffered vasospasm during the hospital course. Most patients, except one who died, had few complications. Four patients had a history of hypertension or were taking medication for hypertension. One patient had autosomal-dominant polycystic kidney disease, but her relative had no such disorder. The main relationship identified in the familial group was that of siblings (brother and brother or sister and sister). Fig. 1 shows the pedigrees

Table 1. Clinical summary of patients with SAH

\begin{tabular}{lcc}
\hline Characteristic & Sporadic Group & Familial Group \\
\hline Number of patients & 1116 & 12 \\
Sex (male/female) & $362 / 754$ & $7 / 5$ \\
Age in years, mean & 54.9 & 49.8 \\
Male & 48.9 & 48.7 \\
Female & 57.8 & 51.2 \\
Aneurysm location (\%) & & \\
A-com & $371(33)$ & $1(8)$ \\
ACA & $52(5)$ & 0 \\
MCA & $263(24)$ & $5(42)$ \\
ICA & $203(18)$ & $3(25)$ \\
Post. circulation & $36(3)$ & 0 \\
Multiple & $191(17)$ & $3(25)$ \\
Aneurysm size (no) & & 9 \\
Small (<10 mm) & 805 & 2 \\
Large (10-24 mm) & 205 & 0 \\
\hline
\end{tabular}

A-com : anterior communicating artery, ACA : anterior cerebral artery, ICA : internal carotid artery, MCA : middle cerebral artery, Multiple : multiple aneurysm, Post. circulation : posterior circulation 


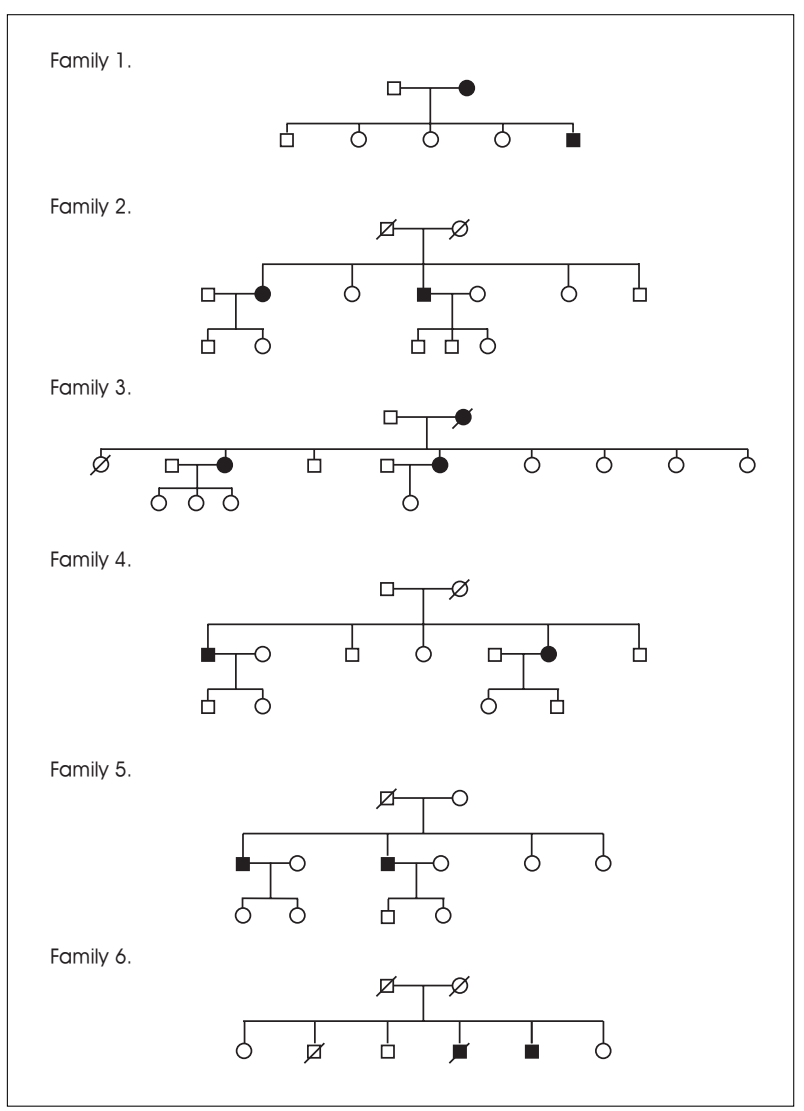

Fig. 1. Pedigrees in the familial group. Most families had affected siblings. We excluded the mother in family 3 because we could not confirm the imaging results and knew of her subarachnoid hemorrhage only from statements by family members, as she received treatment at another hospital.

of the familial group. All patients were first-degree relatives, represented by two siblings in most families.

\section{DISCUSSION}

Many studies have compared the clinical and radiographic characteristics of familial versus sporadic aneurysms, and several distinctive features of familial aneurysms have been identified. Those studies have consistently shown that familial aneurysms rupture earlier and are smaller in size compared with sporadic aneurysms $s^{3,4,10,19)}$. In siblings, aneurysms more commonly rupture within the same decade of life ${ }^{7)}$. Aneurysms in the anterior communicating artery complex are underrepresented in familial aneurysms $\mathrm{s}^{4,19)}$. Multiple aneurysms and de novo aneurysms are more often found in patients with familial aneurysms. Our study is largely consistent with those earlier reports on familial intracranial aneurysms. In our patients, the mean age at the time of rupture in the familial group (49.75 years) was younger than that in the sporadic group (54.9 years), and these ages are similar to those in other studies, which have reported the age of familial aneurysm rupture as 42.3 years, versus 50-54 years for sporadic aneurysms $s^{4,8}$. In our study, as in previous studies, familial aneurysms were less common in the anterior communicating artery and more common in the middle cerebral artery. In the sporadic group, aneurysms occurred most commonly in the anterior communicating artery (371/1,116 cases, 33\%). In contrast, only one of the 12 patients in the familial group had an anterior communicating artery aneurysm. Five families had affected siblings. Table 2 shows the relationship between family members and the age at aneurysm rupture. Except in family 6, familial aneurysms of siblings ruptured within 5 years of each other, during the same decade of life, although the ages differed among families. This finding is consistent with previous studies ${ }^{7}$.

Several aspects of our results were differed from those of previous studies. A higher incidence of familial aneurysms in women has often been reported ${ }^{8,9,19)}$, but our results showed no significant gender difference. Furthermore, the incidence of familial aneurysms in our study was very low $(1.06 \%, 12$ of 1,128 cases). According to previous epidemiological studies on the frequency of familial intracranial aneurysms, $7-20 \%$ of patients with aneurysmal SAH had first- or second-degree relatives with intracranial aneurysms ${ }^{11,14,15)}$.

Our study method had several limitations. We could not screen all of the relatives of SAH patients or asymptomatic relatives and could not identify all relatives treated at other hospitals. We had to rely on interviews for the family histories of relatives. The number of patients treated for $\mathrm{SAH}$ at our hospital was limited. If we were to screen for asymptomatic relatives of SAH patients and relatives living in other communities, the incidence of familial aneurysms would likely be higher.

We raise question of whether there is a need to screen relatives of those with SAH. With the advent of magnetic resonance angiography and three-dimensional computed tomography, noninvasive and minimally invasive screening tools are now available. Although the benefits and risks of screening and the associated costs have not been properly assessed, this important issue has recently been discussed ${ }^{9,12,16}$.

It has been suggested by many authors that members of families in which two or more individuals have a cerebral aneurysm should be screened ${ }^{1,3,9)}$. This rationale was based on prevalence, risk of rupture, and associated mortality and morbidity in familial intracranial aneurysm. The outcome of SAH from a ruptured aneurysm remains poor, with a case fatality rate of $50 \%$ and a rate of functional dependence of $20 \%{ }^{6}$. Moreover, the risk for poor outcome in patients with familial SAH is 1.8 to 2.5 times that in patients with sporadic $\mathrm{SAH}^{3)}$. In addition, first-degree relatives of patients 
Table 2. Characteristics of the familial group

\begin{tabular}{|c|c|c|c|c|c|c|c|c|c|c|}
\hline \multirow{2}{*}{ Family No. } & \multirow{2}{*}{ Relation } & \multirow{2}{*}{ Gender } & \multirow{2}{*}{$\operatorname{Age}^{*}(y)$} & \multicolumn{2}{|c|}{ Location of IA } & \multicolumn{2}{|c|}{ Size of IA (mm) } & \multirow{2}{*}{$\mathrm{H}-\mathrm{HGr}$} & \multirow{2}{*}{ GOS } & \multirow{2}{*}{$\mathrm{PMHX}$} \\
\hline & & & & Ruptured & Unruptured & Ruptured & Unruptured & & & \\
\hline \multirow[t]{2}{*}{1} & Mother & $\mathrm{F}$ & 62 & $\mathrm{MCA}$ & & 4 & & 2 & 5 & HTN, Abd. aortic an. \\
\hline & Son & M & 33 & $\mathrm{~A}-\mathrm{COM}$ & both P-COM & 10 & 4,3 & 3 & 5 & \\
\hline \multirow[t]{2}{*}{2} & Sibling & M & 53 & Ant. choroidal a. & & 8 & & 2 & 5 & \\
\hline & Sibling & $\mathrm{F}$ & 50 & $\mathrm{MCA}$ & & 5 & & 2 & 5 & \\
\hline \multirow[t]{2}{*}{3} & Sibling & $\mathrm{F}$ & 53 & $\mathrm{~A}-\mathrm{COM}$ & & 4 & & 2 & 4 & ADPK, HTN \\
\hline & Sibling & $\mathrm{F}$ & 50 & $\mathrm{P}-\mathrm{COM}$ & & 2 & & 3 & 4 & \\
\hline \multirow[t]{2}{*}{4} & Sibling & $\mathrm{F}$ & 41 & $\mathrm{P}-\mathrm{COM}$ & & 7 & & 2 & 5 & Hypothyroidism \\
\hline & Sibling & $M$ & 39 & $\mathrm{MCA}$ & Opthalmic a. & 3 & 3 & 2 & 5 & \\
\hline \multirow[t]{2}{*}{5} & Sibling & M & 47 & MCA & & 8 & & 2 & 5 & \\
\hline & Sibling & M & 46 & MCA & & & & 2 & 5 & \\
\hline \multirow[t]{3}{*}{6} & Sibling & M & 55 & ACA & $\mathrm{A}-\mathrm{COM}$ & 6 & 3,3 & 3 & 5 & HTN \\
\hline & & & & & $\mathrm{P}-\mathrm{COM}$ & & & & & \\
\hline & Sibling & $M$ & 68 & MCA & & 25 & & 5 & 1 & HTN \\
\hline
\end{tabular}

${ }^{*}$ Age at the time of aneurysm rupture. Abd. aortic an : abdominal aortic aneurysm, A-COM : anterior communicating artery, ACA : anterior cerebral artery, ADPK : autosomal dominant polycystic kidney disease, Ant. choroidal a : anterior choroidal artery, GOS : Glasgow Outcome Scale, HTN : hypertension, H-H Gr : Hunt-Hess grade, IA : intracranial aneurysm, MCA : middle cerebral artery, Ophthalmic a : ophthalmic artery, P-COM : posterior communicating artery, PMHX: past medical history

with sporadic SAH have a 3- to 7-fold increased risk of $\mathrm{SAH}$, and the risk may be even higher in first-degree relatives of patients with familial $\mathrm{SAH}^{2,11}$. Although there are also risks associated with angiography, it may be beneficial to screen relatives of familial SAH patients because of the high risk of death and morbidity, and the risk of early and small-size rupture9). Leblanc et al. have suggested that the best age range for angiographic screening and surgical intervention is from 20 to 49 years for men and 20 to 53.5 years for women, given a respective life expectancy of 32 years based of the poor outcome, high prevalence, and rupture at younger age and smaller size in familial $\mathrm{SAH}^{9}$. However, implementation of a screening program for the first-degree relatives of patients with sporadic SAH does not seem warranted at this time, because the resulting slight increase in life expectancy likely does not offset the risk of postoperative sequelae ${ }^{12}$. Raaymakers et al. have suggested that siblings of familial SAH patients could benefit most from screening programs, because unruptured aneurysms were discovered more often in siblings than in children of patients with SAH; in that study, nine of the 10 relatives with newly discovered aneurysms were the brother or sister of an affected patient ${ }^{15)}$. Marieke et al. found that the yield of repeated screening for familial intracranial aneurysms was high; new aneurysms were detected in $16 \%$ of the relatives with previous aneurysms and in $7 \%$ of the relatives without previous aneurysms, mostly within 5 years ${ }^{21)}$. Rinkel has suggested that because the most important unchangeable risk factors are familial occurrence of SAH and autosomal dominant polycystic kidney disease, individuals with these risk factors should be screened ${ }^{17}$.

In counseling relatives of SAH patients, we keep in mind that the experience of SAH-related death or disability in close relatives might have had an impact on the quality of life of asymptomatic relatives. Anxiety was seen in relatives of familial SAH patients during our study, despite good results, and thus we screened all members of family 3 . The results were negative, and the family members were satisfied. We must also remember that knowledge of the presence of an unruptured aneurysm is associated with a decreased quality of life ${ }^{22}$.

\section{CONCLUSION}

Although the small number of patients and the local geographic setting are limitations to this study, we suggest that family history is important when caring for SAH patients and their relatives. We examined familial intracranial aneurysms, and our results were similar to those of previous studies, except for gender differences and incidence. Compared with sporadic aneurysms, familial aneurysms ruptured at a younger age and smaller size. Familial aneurysms occurred frequently in the middle cerebral artery and were underrepresented in the anterior communicating artery. Based on the results of this and previous studies, screening should be considered in the fifth or sixth decade for those who have a sibling with SAH.

\section{References}

1. Broderick JP, Sauerbeck LR, Foroud T, Huston J, Pankratz N, Meissner I, et al : The familial intracranial aneurysm (FIA) study protocol. BMC Medical Genet $6: 17,2005$

2. Bromberg JE, Rinkel GJ, Algra A, Greebe P, van Duyn CM, Hasan $\mathrm{D}$, et al. : Subarachnoid hemorrhage in first and second degree relatives of patients with subarachnoid hemorrhage. BMJ 311 : 288289,1995 
3. Bromberg JE, Rinkel GJ, Algra a, Limburg M, van Gijn J : Outcome in familial subarachnoid hemorrhage. Stroke 26 : 961-963, 1995

4. Bromberg JE, Rinkel GJ, Algra A, van Duyn CM, Greebe P, Ramos $\mathrm{LM}$, et al : Familial subarachnoid hemorrhage : distinctive features and patterns of inheritance. Ann Neurol 38 : 929-934, 1995

5. Chambers WR, Harper BJ Jr, Simpson JR : Familial incidence of congenital aneurysms of cerebral arteries. Report of cases of ruptured aneurysms in father and son. J Am Med Assoc 155 : 358-359, 1954

6. Hop JW, Rinkel GJ, Algra A, van Gijn J : Case-fatality rates and functional outcome after subarachnoid hemorrhage : a systematic review. Stroke 28 : 660-664, 1997

7. Kasuya H, Onda H, Takeshita M, Hori T, Takakura K : Clinical features of intracranial aneurysms in siblings. Neurosurgery 46 : 1301-1305; discussion 1305-1306, 2000

8. Leblanc R : Familial cerebral aneurysms. Can J Neurol Sci 24 : 191199, 1997

9. Leblanc R, Worsley KJ, Melanson D, Tampieri D : Angiographic screening and elective surgery of familial cerebral aneurysms : a decision analysis. Neurosurgery 35 : 9-18; discussion 18-19, 1994

10. Lindgaard L, Eskesen V, Gjerris F, Olsen NV : Familial aggregation of intracranial aneurysms in an Inuit patient population in Kalaallit Nunaat (Greenland). Neurosurgery 52 : 357-362; discussion 362363, 2003

11. Lozano AM, Leblanc R : Familial intracranial aneurysms. J Neurosurg $66: 522-528,1987$

12. Magnetic Resonance Angiography in Relatives of Patients with Subarachnoid Haemorrhage (MARS) Study Group : Risk and benefits of screenning for intracranial aneurysms in first-degree relatives of patients with sporadic subarachnoid hemorrhage. $\mathrm{N}$ Engl J Med 341 : 1344-1350, 1999

13. Park JK, Kim HJ, Chang SJ, Koh SB, Koh SY : Risk factors for hem- orrhagic stroke in Wonju, Korea. Yonsei Med J 39 : 229-235, 1998

14. Raaymaker TWM, MARS Study Group : Aneurysms in relatives of patients with subarachnoid hemorrhage : frequency and risk factors. Neurology 53 : 982-988, 1999

15. Raaymakers TW, Rinkel GJ, Ramos LM : Initial and follow-up screening for aneurysms in families with familial subarachnoid hemorrhage. Neurology 51 : 1125-1130, 1998

16. Rinkel GJ : Intracranial aneurysm screening : indications and advice for practice. Lancet Neurol $4: 122-128,2005$

17. Ruigrok YM, Rinkel GJ, Algra A, Raaymakers TW, van Gijn J : Characteristics of intracranial aneurysms in patients with familial subarachnoid hemorrhage. Neurology $62: 891-894,2004$

18. Teasdale GM, Wardlaw JM, White PM, Murray G, Teasdale EM, Easton $\mathrm{V}$ : The familial risk of subarachnoid haemorrhage. Brain $128: 1677-1685,2005$

19. ter Berg HW, Dippel DW, Limburg M, Schievink WI, van Gijn J : Familial intracranial aneurysms. A review. Stroke 23 : 1024-1030, 1992

20. Teunissen LL, Rinkel GJ, Algra A, van Gijn J : Risk factors for subarachnoid hemorrhage. Stroke $27: 544-549,1996$

21. Wermer MJ, Rinkel GJ, van Gijn J : Repeated screening for intracranial aneurysms in familial subarachnoid hemorrhage. Stroke $34: 2788-2791,2003$

22. Wermer MJ, van der Schaaf IC, Van Nunen P, Bossuyt PM, Anderson CS, Rinkel GJ : Psychosocial impact of screening for intracranial aneurysms in relatives with familial subarachnoid hemorrhage. Stroke $36: 836-840,2005$

23. Wills S, Ronkainen A, van der Voet M, Kuivaniemi H, Helin K, Leinonen E, et al : Familial intracranial aneurysm: an analysis of 346 multiplex Finnish families. Stroke 34 : 1370-1374, 2003 